\title{
El tratamiento con sibutramina es efectivo para la reducción de peso a largo plazo
}

Long - term weight loss with sibutramine. Wirth A, Krause J. Jama 2001;286:1331-1339.

\section{Objetivo}

Comparar la eficacia de dos tratamientos con sibutramina para la reducción de peso en obesos.

Diseño

Ensayo clínico randomizado doble ciego y controlado con placebo.Seguimiento 48 semanas.

\section{Lugar}

Estudio multicéntrico realizado en consultorios privados y consultorios externos de hospitales universitarios de Inglaterra.

\section{Pacientes}

Se estudiaron 1.102 pacientes de ambos sexos, entre 18 a 65 años y obesos con un índice de masa corporal (IMC) de 30 a 40 $\mathrm{kg} / \mathrm{m} 2$.

\section{Intervención}

Durante cuatro semanas los pacientes recibieron $15 \mathrm{mg}$ de sibutramina. Los que redujeron al menos $2 \%$ del peso y $/ 02 \mathrm{~kg} .0$ más (1.001 pacientes) fueron randomizados en relación 2:2:1 y tratados durante 44 semanas. Un grupo recibió tratamiento continuo con $15 \mathrm{mg}$ de sibutramina diarios, otro recibió tratamiento con 15 $\mathrm{mg}$ de sibutramina en forma intermitente durante las semanas 1 a 12,19 a 30 y 37 a 48 y placebo el resto de las semanas; otro grupo recibió sólo placebo. No se les realizó programa dietético ni modificación de la conducta. Tanto los pacientes como el investigador fueron ciegos al tratamiento.

\section{Medición del resultado principal}

Se comparó la pérdida de peso en los tres grupos en el período de tratamiento. Secundariamente se estudiaron la circunferencia de la cintura, tensión arterial, trigliceridemia, colesterol total, HDLc, LDLc.

\section{Resultados}

Durante las cuatro primeras semanas la pérdida de peso fue similar en los tres grupos: $4.1 \mathrm{~kg}(4.2 \%), 4.5 \mathrm{~kg}(4.6 \%)$ y $4 \mathrm{~kg}(4.1 \%)$ en tratamiento continuo, intermitente y placebo respectivamente. Luego de la aleatorización, el grupo con tratamiento continuo tuvo una reducción de $4 \%$ del peso (3.8 kg, IC 95\% 4.42 - 3.20); los que recibieron tratamiento intermitente perdieron $3.5 \%(3.3 \mathrm{~kg}$, IC $95 \% 3.96-2.66$ ) y los que recibieron placebo aumentaron $0.2 \%$. La pérdida de peso de los grupos de tratamiento continuo o intermitente con sibutramina comparado con el grupo que recibió placebo fue estadísticamente significativa $(p<0.001)$ Al comparar los dos grupos con sibutramina, si bien el grupo que la recibió en forma continua tuvo una mayor pérdida de peso, la diferencia no fue estadísticamente significativa $(p=0.28)$. La pérdida total de peso durante las 48 semanas fue de $7.9 \mathrm{~kg}$; en los grupos continuo e intermitente y $3.8 \mathrm{~kg}$ en el grupo placebo.Cabe destacar que la pérdida de peso en el grupo placebo fue durante las 4 semanas iniciales por lo que se le puede atribuir netamente al efecto de la sibutramina.

Perdieron el $5 \%$ del peso corporal el $65 \%$ de los pacientes con tratamiento continuo, el $63 \%$ con tratamiento intermitente y el $35 \%$ de los que tenían placebo. El $10 \%$ de pérdida lo experimentaron el $32 \%$ en tratamiento continuo, el $33 \%$ con intermitente y el $13 \%$ de los que recibieron placebo.La pérdida de peso en los tratamientos activos fue significativamente mayor que en el grupo placebo tanto para el 5 como para el $10 \%$ de pérdida $(p<0.001)$.Los dos grupos con sibutramina no mostraron diferencias significativas $(p=0.22$ para el $5 \%$ y $p=0.39$ para el $10 \%)$

Los tres grupos presentaron reducción de la circunferencia de la cintura, del nivel de LDLc y triglicéridos; incremento de la media de HDLc y no se observó cambio en el colesterol total y la tensión arterial.

\section{Conclusiones}

El tratamiento con sibutramina es eficaz para la reducción de peso y la consecuente mejora de los factores de riesgo cardiovascular.

Fuente de Financiamiento:No referida

\section{Comentario}

La obesidad es una enfermedad seria y crónica con una variedad de causas subyacentes y condiciones asociadas que conducen a mayor morbilidad y mortalidad. Este estudio demostró que los pacientes que reciben sibutramina tienen una significativa pérdida de peso.Estos resultados confirman estudios previos realizados 1, 2, 3 . La disminución del 5 al $10 \%$ del peso corporal genera beneficios clínicos asociados con la disminución de factores de riesgo cardiovascular.La pérdida de peso trae aparejado la disminución de la tensión arterial, triglicéridos, colesterol total, HDLc, LDLc. En este estudio, más del $60 \%$ de los pacientes en los dos tratamientos activos perdió el $5 \%$ o más del peso comparado con el $35 \%$ de los que recibieron placebo. Cabe destacar que la disminución de los factores de riesgo cardiovascular no son específicos de la droga sino del resultado de la disminución de peso.

Un pequeño número de pacientes abandonó el tratamiento. Estudios previos han demostrado que los que no pierden al menos el $2 \%$ del peso en las primeras semanas no se benefician con el tratamiento a largo plazo 4

Aún con estos resultados, ningún fármaco en forma exclusiva podrá dar solución al problema de la obesidad.En los pacientes con obesidad moderada y severa la farmacología deberá ser incluida como parte integrante de una estrategia de tratamiento crónico conjuntamente con un plan de alimentación hipocalórico, terapia psicológica y actividad física para lograr resultados a largo plazo.

Dra. Sonia Lombardelli [ Unidad de Nutrición Especial.Servicio de Diabetología y Nutnición. Hospital Privado de Córdoba ]

\footnotetext{
Referencias

1.Jones SP, Smith IG, Kelly F, Gray JA.Long term weight loss with sibutramine.Int J Obes Relat Metab Disord.1995;19:41.

2.Apfelbaum M, Vague P, Ziegler O, Hanolin C, Thomas F, Leutnegger E.Long term maintenance of weigth loss after a very low calorie diet:a randomized blinded trial of the efficacy and tolerability of sibutramine.Am J Med, 1999;106:179-184

3.James WP, Astrup A, Finer N, et al, for de STORM Study Group.Effect of sibutramine on weight maintenance after weigth loss:a randomized trial.Lancet.2000;356:2119-2125. 4.Bray GA, Blackburn GL, Ferguson JM, et al.Sibutramine produces dose related weigth loss.Obes Res 1999;7:189-198.
} 